\title{
Nonlinear Observer-based Fault Detection and Isolation for Wind Turbines
}

\author{
Abdulhamed Hwas and Reza Katebi \\ Industrial Control Centre, University of Strathclyde, Glasgow, UK \\ Email: Abdulhamed.hwas@strath.ac.uk,r.katebi@eee.strath.ac.uk
}

\begin{abstract}
This paper is concerned with the development of a novel nonlinear observer-based scheme for early Fault Detection and Isolation (FDI) in wind turbines. The method is based on designing a nonlinear observer using State Dependent Differential Riccati Equation (SDDRE) and a nonlinear model of the 5MW wind turbine. The fault detection system is designed and optimized to be most sensitive to system faults and least sensitive to system disturbances and noises. The comparison of system outputs with nonlinear observer outputs are given to demonstrate good estimation performance. The residual generator based on the nonlinear observer is also employed to develop a monitoring system. Simulation results presented to illustrate that the proposed method is robust and can detect and isolate a fault or multi-faults in sensors of the wind turbine.
\end{abstract}

Keywords- fault detection and isolation; nonlinear observer; wind turbine; state dependent differential riccati equation

Nomenclature:

$\begin{array}{cl}J_{T} & \text { Turbine inertia } \\ J_{G} & \text { Generator inertia } \\ K_{s} & \text { Torsional stiffness } \\ C_{s} & \text { Torsional damping } \\ \omega_{s} & \text { Synchronous speed } \\ R_{s} & \text { Stator resistance } \\ R_{r} & \text { Rotor resistance } \\ L_{s} & \text { Stator inductance } \\ \omega_{w t} & \text { Wind turbine speed }\end{array}$

$\begin{array}{cl}n_{g} & \text { Gearbox ratio } \\ n_{p} & \text { Pole pairs } \\ \beta & \text { Pitch angle } \\ T_{w t} & \text { Mechanical torque } \\ T_{e} & \text { Electrical torque } \\ V_{s} & \text { Stator voltage } \\ L_{m} & \text { Mutual inductance } \\ L_{r} & \text { Rotor inductance } \\ \omega_{m} & \text { Generator speed }\end{array}$

\section{INTRODUCTION}

A nonlinear observer is a dynamic filter which estimates the states or outputs of the system based on a mathematical model, sensor measurements and input commands. In [1], a linear observer is designed and applied for the case where the rotor speed varies slowly. However, in the real case, wind speed and a generator's rotor speed are both variables, and the behaviour of a wind turbine is nonlinear, which should be considered in the FDI design.

This paper is aimed at detecting faults in wind turbine's sensors. These include pitch angle, rotor angle, generator rotor angle, wind turbine rotor speed, generator rotor speed, electric torque; and wind turbine torque faults.

This paper presents a framework for designing a nonlinear observer using state dependent differential Riccati equation rather than algebraic Riccati equations, which can require an overly restrictive requirement on the observability and controllability of the system [2]. The SDDRE, based on extended linearization of the process dynamics, and a solution of SDDRE can be solved by using numerical integration methods.

The paper is organized as follows: Nonlinear model for 5 MW wind turbine is presented in next section. Nonlinear observer is designed and tested in section III and IV. Nonlinear observer-based FDI scheme is briefly described in section V. Sensor fault detection and isolation scheme and simulation results are demonstrated in section VI and VII, respectively. Finally, the conclusion is drawn in section VIII.

\section{NONLINEAR MODEL FOR A 5 MW WIND TURBINE}

Based on the wind turbine in [3] the differential equations for the subsystems are:

\section{A. Actuator model}

The pitch actuator consists of a mechanical and a hydraulic system, which is used to turn the blades along their longitudinal axis. The actuator model describes the dynamic behaviour between a pitch demand $\beta_{d}$ from the pitch controller and the measurement of a pitch angle $\beta$. The dynamics of the blades are non-linear with saturation limits on both pitch angle and pitch rate. This saturation is caused by high frequency components of the pitch demand spectrum, via measurement noise, and spectral peaks induced by rotational sampling [4]. In this paper, the constraint is not considered. The actuator dynamic is modelled as in [5]. The change in the pitch angle is:

$$
\dot{\beta}=\frac{1}{\tau_{\beta}}\left(\beta_{d}-\beta\right)
$$

where $\tau_{\beta}$ is a time constant of the pitch actuator.

\section{B. Two-mass drive-train model}

When compared with other mechanical models of the wind turbine, our first concern is the dynamic model of the drivetrain. The reason is to give priority to the parts of the dynamic structure of the wind turbine which contributed to grid integration $[6,7]$. Often, a two-mass drive-train model is applied when analysing the interaction of the wind turbine with the grid. Therefore, the drive train has a significant influence on the power fluctuations [8]. In addition, the torque control can assist in dampening the mechanical oscillations. A two-mass drive-train can be described as a mathematical model in equation (2). 


$$
\begin{aligned}
& \dot{\omega}_{w t}=\frac{1}{J_{T}}\left(T_{w t}-K_{s} \theta_{K}-C_{s} \omega_{w t}+\frac{C_{s}}{n_{g}} \omega_{m}\right) \\
& \dot{\omega}_{m}=\frac{1}{J_{G}}\left(\frac{K_{s}}{n_{g}} \theta_{K}+\frac{C_{s}}{n_{g}} \omega_{w t}-\frac{C_{s}}{n_{g}^{2}} \omega_{m}-T_{e}^{c}-b \omega_{m}\right)
\end{aligned}
$$

where $T_{e}^{c}$ is a control signal for electrical torque. $b$ is damping coefficient. The difference between turbine rotor angle and generator rotor angle $\left(\theta_{K}\right)$ is:

$$
\dot{\theta}_{k}=\omega_{w t}-\frac{\omega_{m}}{n_{g}}
$$

\section{Doubly fed induction generator model}

State equations of the $4^{\text {th }}$ order doubly fed induction generator model can be expressed in $\mathrm{d}$, $\mathrm{q}$ frame as [13]:

$$
\begin{aligned}
& \dot{\psi}_{d s}=-R_{s} i_{d s}+\omega_{s} L_{s} i_{q s}+\omega_{s} L_{m} i_{q r}+v_{d s} \\
& \dot{\psi}_{q s}=-\omega_{s} L_{s} i_{d s}-R_{s} i_{q s}-\omega_{s} L_{m} i_{d r}+v_{q s} \\
& \dot{\psi}_{d r}=\omega_{s} L_{m} i_{q s}-R_{r} i_{d r}+\omega_{s} L_{r} i_{q r}-\omega_{m} L_{m} i_{q s}-\omega_{m} L_{r} i_{q r}+v_{d r} \\
& \dot{\psi}_{q r}=-\omega_{s} L_{m} i_{d s}-\omega_{s} L_{r} i_{d r}-R_{r} i_{q r}+\omega_{m} L_{m} i_{d s}+\omega_{m} L_{r} i_{d r}+v_{q r}
\end{aligned}
$$

where $\dot{\psi}_{d s}, \dot{\psi}_{q s}, \dot{\psi}_{d r}, \dot{\psi}_{q r}, i_{d s}, i_{q s}, i_{d r}, i_{q r}, v_{d s}, v_{q s}, v_{d r}, v_{q r}$ are stator and rotor flux, current and voltage are expressed by the $\mathrm{d}$ axis and q-axis components.

By computing the observability matrix for state-space model, for an $n$-by- $n$ matrix $A$ and a $r$-by- $n$ matrix $C$,

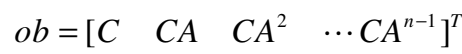

where $n$ and $r$ are dimensions of state and output.

The system described in equation (1) to (4) is not completely observable. Therefore, we need to revise the model. The order of the DFIG model can be modified by assuming:

- The stator magnetizing current space is phasor $\vec{i}_{m s}=\left|\vec{i}_{m s}\right|$. In the steady state, it is $\left|\vec{i}_{m s}\right|=$ constant,

- The frequency of the power supply to the stator is constant ( $f=50 \mathrm{~Hz}$ ) and, consequently, $\omega_{s}=\frac{2 \pi f}{n_{p}}$.

- The stator resistance $R_{s}$ can be disregarded (usually acceptable where a generator power is very high).

Then, from stator and rotor flux $\psi$ equation (4) can be rewritten as [9]:

$$
\begin{aligned}
& \psi_{d s}=L_{m}\left|\vec{i}_{m s}\right|, \psi_{q s}=L_{s} i_{q s}+L_{m} i_{q r}=0 \\
& \psi_{d r}=L_{m}^{2}\left|\vec{i}_{m s}\right|+\sigma L_{r} i_{d r}, \psi_{q r}=\sigma L_{r} i_{q r}
\end{aligned}
$$

where $\left|\vec{i}_{m s}\right|=\frac{\left|\vec{v}_{s}\right|}{\omega_{s} L_{m}}, \quad$ leakage coefficient $\sigma=1-\frac{L_{m}^{2}}{L_{s} L_{r}}$ and $\left|\vec{v}_{s}\right|=\sqrt{3} V_{s} . V s$ is the stator voltage.

State equations of the $4^{\text {th }}$ order DFIG model (4) after some substitutions can be written as [3]:

$$
\begin{aligned}
& v_{d s}=R_{s} i_{d s}-\omega_{s} \psi_{q s}+\frac{d \psi_{d s}}{d t} \approx 0 \\
& v_{q s}=R_{s} i_{q s}+\omega_{s} \psi_{d s}+\frac{d \psi_{q s}}{d t} \approx \omega_{s} \psi_{d s}=\left|\vec{v}_{s}\right| \\
& v_{d r}=\sigma L_{r} \frac{d i_{d r}}{d t}+R_{r} i_{d r}-\left(\omega_{s}-\omega_{m}\right) \sigma L_{r} i_{q r} \\
& v_{q r}=\sigma L_{r} \frac{d i_{q r}}{d t}+R_{r} i_{q r}+\left(\omega_{s}-\omega_{m}\right)\left(\sigma L_{r} i_{d r}+\frac{\sqrt{3} L_{m} V_{s}}{\omega_{s} L_{s}}\right)
\end{aligned}
$$

The nonlinear model of a $5 \mathrm{MW}$ wind turbine can now be written as:

$$
\dot{x}=f(x, u), \quad y=g(x, u)
$$

where

$$
\dot{x}=\left[\begin{array}{l}
-\frac{1}{\tau_{\beta}} \mathrm{x}_{1}+\frac{1}{\tau_{\beta}} \mathrm{u}_{1} \\
\mathrm{x}_{3}-\frac{1}{n_{g}} \mathrm{x}_{4} \\
-\frac{K_{s}}{J_{T}} \mathrm{x}_{2}-\frac{K_{s}}{J_{T}} \mathrm{x}_{3}+\frac{C_{s}}{J_{T} n_{g}} \mathrm{x}_{4}+\frac{1}{J_{T}} \mathrm{u}_{2} \\
\frac{K_{s}}{J_{G} n_{g}} \mathrm{x}_{2}+\frac{C_{s}}{J_{G} n_{g}} \mathrm{x}_{3}-\left(\frac{C_{s}}{J_{G} n_{g}^{2}}+b_{d}\right) \mathrm{x}_{4}-\frac{1}{J_{G}} \mathrm{u}_{3} \\
-\mathrm{x}_{6} \mathrm{x}_{4}-\frac{R_{r}}{\sigma L_{r}} \mathrm{x}_{5}+\omega_{s} \mathrm{x}_{6}+\frac{1}{\sigma L_{r}} \mathrm{u}_{4} \\
\frac{\sqrt{3} L_{m} V_{s}}{\sigma L_{s} L_{r} \omega_{s}} \mathrm{x}_{4}+\left(\mathrm{x}_{4}-\omega_{s}\right) \mathrm{x}_{5}-\frac{R_{r}}{\sigma L_{r}} \mathrm{x}_{6}+\frac{1}{\sigma L_{r}} u_{5}-\frac{\sqrt{3} L_{m} V_{s}}{\sigma L_{s} L_{r}}
\end{array}\right]
$$

The output equation is:

$y=\left[x_{1} ; x_{2} ; \quad x_{3} ; x_{4} ; K_{s} x_{2}+C_{s} x_{3}-\frac{C_{s}}{n_{g}} x_{44} ; \frac{n_{p} \sqrt{3} L_{m} V_{s}}{L_{s} \omega_{s}} x_{6}\right]^{T}$

The state vector, input vector and output are defined as:

$$
\begin{aligned}
x & =\left[\begin{array}{llllll}
\beta & \theta_{k} & \omega_{w t} & \omega_{m} & i_{d r} & i_{q r}
\end{array}\right]^{T} \\
u & =\left[\begin{array}{llllll}
\beta_{d} & T_{w t} & T_{e}^{c} & v_{d r} & v_{q r}
\end{array}\right]^{T} \\
y & =\left[\begin{array}{llllll}
\beta & \theta_{k} & \omega_{w t} & \omega_{m} & T_{w t} & T_{e}
\end{array}\right]^{T} \\
& \text { Nonlinear observer design }
\end{aligned}
$$

The nonlinear dynamic model (9) can be represented by the following structure having state-dependent coefficients:

$$
\dot{x}=A(x) x+B(u) u, \quad y=C x
$$

where:

$$
A(x)=\left[\begin{array}{cccccc}
-\frac{1}{\tau_{\beta}} & 0 & 0 & 0 & 0 & 0 \\
0 & 0 & 1 & -\frac{1}{n_{g}} & 0 & 0 \\
0 & -\frac{K_{s}}{J_{T}} & -\frac{K_{s}}{J_{T}} & \frac{C_{s}}{J_{T} n_{g}} & 0 & 0 \\
0 & \frac{K_{s}}{J_{G} n_{g}} & \frac{C_{s}}{J_{G} n_{g}} & -\left(\frac{C_{s}}{J_{g} n_{g}^{2}}+b_{d}\right) & 0 & 0 \\
0 & 0 & 0 & -\mathrm{x}_{6} & -\frac{R_{r}}{\sigma L_{r}} & \omega_{s} \\
0 & 0 & 0 & \frac{\sqrt{3} L_{m} V_{s}}{\sigma L_{s} L_{r} \omega_{s}} & \left(\mathrm{x}_{4}-\omega_{s}\right) & -\frac{R_{r}}{\sigma L_{r}}
\end{array}\right]
$$




$$
\begin{aligned}
B(u) & =\left[\begin{array}{ccccc}
\frac{1}{\tau_{\beta}} & 0 & 0 & 0 & 0 \\
0 & 0 & 0 & 0 & 0 \\
0 & \frac{1}{J_{T}} & 0 & 0 & 0 \\
0 & 0 & -\frac{1}{J_{G}} & 0 & 0 \\
0 & 0 & 0 & \frac{1}{\sigma L_{r}} & 0 \\
0 & 0 & 0 & 0 & \frac{1}{\sigma L_{r}}-\frac{\sqrt{3} L_{m} V_{s}}{\sigma L_{s} L_{r} u_{5}}
\end{array}\right] \\
C & =\left[\begin{array}{cccccc}
1 & 0 & 0 & 0 & 0 & 0 \\
0 & 1 & 0 & 0 & 0 & 0 \\
0 & 0 & 1 & 0 & 0 & 0 \\
0 & 0 & 0 & 1 & 0 & 0 \\
0 & K_{s} & C_{s} & -\frac{C_{s}}{n_{g}} & 0 & 0 \\
0 & 0 & 0 & 0 & 0 & \frac{n_{p} \sqrt{3} L_{m} V_{s}}{L_{s} \omega_{s}}
\end{array}\right]
\end{aligned}
$$

After bringing the system to the states dependent coefficient form as in equation (12), we constructed an observer which has the same structure as equation (12) plus the driving feedback term whose role is to reduce the observation error to zero, i.e. as:

$$
\dot{\hat{x}}=A(\hat{x}) \hat{x}+B(u) u+K(\hat{x})[y-C \hat{x}]
$$

The nonlinear observer gain matrix in equation (17) is solved using the Riccati differential equation (18) to find matrix $P(\hat{x})$ using $A(x), C$ and $Q=C^{T} C \geq 0$, and $R(x)>0$ for all $x$.

$$
\begin{gathered}
K(\hat{x})=P(\hat{x}) C^{T} R^{-1} \\
\dot{P}(\hat{x})=P(\hat{x}) A^{T}(\hat{x})+A(\hat{x}) P(\hat{x})-P(\hat{x}) C^{T} R^{-1} C P(\hat{x})+Q
\end{gathered}
$$

\section{NONLINEAR OBSERVER SIMULATION RESULTS}

Simulation at different wind speeds from cut-in to cut-out $(3 \mathrm{~m} / \mathrm{s}-25 \mathrm{~m} / \mathrm{s})$ are investigated in order to assess the performance of the nonlinear observer in comparison with the output of the wind turbine. For instance, Fig. 1 shows the simulation results for $R=10^{6}$. These figures show a comparison of system outputs and estimated states. The following sub figures confirm the good performance of the nonlinear observer, (a) Pitch angle; (b) difference between turbine rotor angle and generator rotor angle $\left(\theta_{K}\right)$; (c) wind turbine rotor speed; (d) generator rotor speed; (e) electric torque and (f) wind turbine torque where the effective wind speed is $15 \mathrm{~m} / \mathrm{s}$. sub-figures (b, c, d and e) the outputs are similar. Estimate states values converge rapidly to the nonlinear model output. Therefore, we can conclude that the nonlinear observer match well to estimate states of the wind turbine.
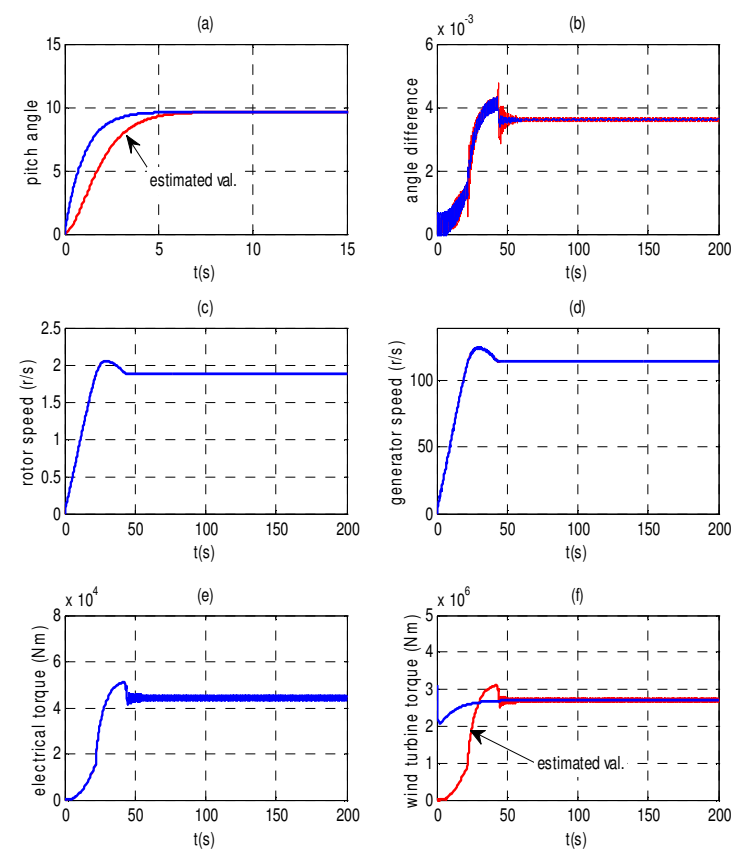

Fig. 1. Comparison of system outputs with nonlinear observer estimation, where the effective wind speed is equal $15 \mathrm{~m} / \mathrm{s}$

\section{NONLINEAR OBSERVER-BASED FDI SCHEME}

The state space model of the wind turbine in the equation (12) can be extended to include faults and disturbances as follows:

$$
\begin{aligned}
& \dot{x}=A(x) x+B(u) u+R_{1} f+d \\
& y=C x+D u+R_{2} f
\end{aligned}
$$

The equation of the observer using this model for $d=f=$ 0, will be:

$$
\begin{aligned}
& \dot{\hat{x}}=A(\hat{x}) \hat{x}+B(u) u+K(\hat{x})[y-C \hat{x}] \\
& \hat{y}=C \hat{x}+D u
\end{aligned}
$$

$f$ is the fault vector which is considered to be an unknown time function. The vector $d$ is the disturbance vector which can be written as:

$$
d=\Delta A(x) x+\Delta B(u) u
$$

The residual generator, studied in this section, is based on a nonlinear observer. It is applied to the monitoring system represented by equation (19). To define the error, equation (20) is subtracted from equation (19) to obtain the estimated error equation (22) and residual equation (23):

$$
\dot{e}=\dot{x}-\dot{\hat{x}}=\left(A(x)-P(\hat{x}) C^{T} R^{-1} C\right) e+d+R_{1} f-P(\hat{x}) C^{T} R^{-1} R_{2} f
$$

and residual equation is:

$$
r=Q[y-\hat{y}]=Q\left[\mathrm{Ce}+R_{2} f\right]
$$

where $Q$ is the residual weighting factor matrix; $R_{1}$ and $R_{2}$ are fault matrices; 
To implement FDI, two steps should be taken. Firstly, sensitive and insensitive relationships between residuals and faults for each type of faults (actuator, sensor, and component faults.) should be defined by rewriting equation (19) in the form as:

$$
\begin{aligned}
& \dot{x}=A(x) x+B(u) u+R_{1}^{a} f^{a}+R_{1}^{s} f^{s}+R_{1}^{c} f^{c}+d \\
& y=C x+D u+R_{2}^{a} f^{a}+R_{2}^{s} f^{s}+R_{2}^{c} f^{c}
\end{aligned}
$$

$a, s$ and $c$ denote actuator, sensor and component, respectively.

Secondly, a set of residuals based on equation (24) should be defined. Faults are classified into three groups of actuator, sensor, and component faults and performance indices are then defined as in equations (25), (26) and (27) as follows [1]:

$$
\begin{gathered}
J_{a f}\left(P, R, Q_{a}\right)=\sup _{a \in\left[a_{0}, \omega_{2}\right]} \bar{\sigma}\left\{-\left[Q_{a} D+Q_{a} C(j \omega I-A(x)+P(x)\right.\right. \\
\left.\left.\left.C^{T} R^{-1} C\right)^{-1}\left(B(u)-P(x) C^{T} R^{-1} D\right)\right]\right\} \\
J_{s f}\left(P, R, Q_{s}\right)=\sup _{\omega \in\left[\omega_{1}, \omega_{2}\right]} \bar{\sigma}\left\{\left[Q_{s} I+Q_{s} C\left(j \omega I-A(x)+P(x) C^{T}\right.\right.\right. \\
\left.\left.\left.R^{-1} C\right)^{-1}\left(-P(x) C^{T} R^{-1} I\right)\right]^{-1}\right\} \\
J_{c f}\left(P, R, Q_{c}\right)=\sup _{\omega \in\left[\omega_{1}, \omega_{2}\right]} \bar{\sigma}\left\{-\left[Q_{c} C(j \omega I-A(x)+P(x)\right.\right. \\
\left.\left.\left.\left.C^{T} R^{-1} C\right)^{-1}\right)\right]\right\} \\
\quad J_{d}(P, R)=\left\|\left(A(x)-P(x) C^{T} R^{-1} C\right)^{-1}\right\|
\end{gathered}
$$

where $J_{a f}\left(P, R, Q_{a}\right), J_{s f}\left(P, R, Q_{s}\right), J_{c f}\left(P, R, Q_{c}\right)$ are actuator, sensor component performance indices and $J_{d}(P, R)$ is a disturbance performance index. $Q a, Q s$ and $Q c$ are the residual weighting factor matrices for the actuator, sensor and component, respectively. $\bar{\sigma}_{\{.\}}$denotes to maximal singular value.

The actuator, sensor and component fault matrices are defined as[1]:

$$
R_{1}=\left\{\begin{array}{c|c}
0 & \text { sensor fault } \\
B(u) & \text { actuator fault } \\
A(x) & \text { component fault }
\end{array}, R_{2}=\left\{\begin{array}{c|c}
C & \text { sensor fault } \\
D & \text { actuator fault } \\
0 & \text { component fault }
\end{array}\right.\right.
$$

From equations (26) and (28), the performance indices $J_{s f}\left(P, R, Q_{s}\right)$ and $J_{d}(P, R)$ are functions of $P(x), R$ and $Q_{s}$. Therefore, these parameters should be optimised for stability of the nonlinear observer and optimised by maximising the effect of the sensor faults and minimising the effect of disturbance [10]. Hence, we have a multi-objective optimisation problem. Firstly, $P(x)$ and $R$, which are represented in the Riccati equation (18), are optimised to find the best nonlinear observer gain matrix $K(\hat{x})$.

The initial matrix $P_{0}$ is assumed to be zero matrix. $P(x)$ is a function of states. Consequently, we can only optimise $R$, which gives better sensitivity to faults.
Secondly, define the residual weighting factor matrix $Q_{s k}$ which gives the appropriate residual dimensions for all output via simulating the simulink model. The values of $Q_{s k}$ are defined in the equation (30).

$$
Q_{s k}=\operatorname{diag}\left[q_{1} \ldots . q_{6}\right]
$$

where $q_{1}=10^{5}, q_{2}=10^{5}, q_{3}=10^{5}, q_{4}=5 \times 10^{2}, q_{5}=10, q_{6}=1$.

\section{NONLINEAR OBSERVER-BASED SENSOR FAULT DETECTION AND ISOLATION SCHEME}

\section{A. Fault model}

Faults are modelled as unknown change in signals as an additive fault. This fault can be classified according to their source as an actuator $\Delta u(t)$, sensor $\Delta y(t)$ or component $\Delta u_{c}(t)$ faults [5]. The component faults affect both the true output $(y(t))$ and the observed output $\left(y_{o}^{c}(\mathrm{t})\right)$. The observed signals for the input and output can be rewritten as below [1].

$$
\begin{gathered}
u_{o}(t)=u(t)+\Delta u(t)+\delta u(t) \\
y_{o}^{c}(t)=u_{o}(t)+\Delta u_{c}(t)+\delta u_{c}(t) \\
y_{o}(t)=y_{0}^{c}(t)+\Delta y(t)+\delta y(t)
\end{gathered}
$$

where $u_{0}(t), y_{0}^{c}(t)$ and $y_{o}(t)$ are the actuator, component and sensor outputs respectively. $\delta u(t)$ and $\delta u_{c}(t)$ are actuator and component disturbance signals, $\delta y(t)$ is a sensor noise signal.

\section{B. Wind turbine control}

Below rated wind speed, optimising the power output of the wind turbine is optimised by using the torque control scheme for a variable-speed wind turbine, which is written as [11]:

$$
T_{e(\text { ref })}=\frac{\mathrm{P}_{\mathrm{wt}(\max )}}{\omega_{m}}=\frac{0.5 \rho \pi R_{w}^{5} C_{p_{-} \max } \omega_{m}^{2}}{\lambda_{o p t}^{3} n_{g}^{3}}
$$

where $T_{e(r e f)}$ is the reference electrical torque, $C_{p_{-} \text {max }}$ is the maximum power coefficient, and $\lambda_{\text {opt }}$ is the tip speed ratio at $C_{p_{\_} \max }$. The torque controller is designed and investigated in [3] By using the Internal Model Control based PI controller.

Above rated wind speed, the rotor power must be kept at below design limits by using the pitch angle controller. Fig. 2 shows the block diagram of the mathematical models for the main components of 5MW wind turbine system, particularly aerodynamic, two-mass drive train and generator. The Pitch angle torque controller is designed and investigated in [12]. 


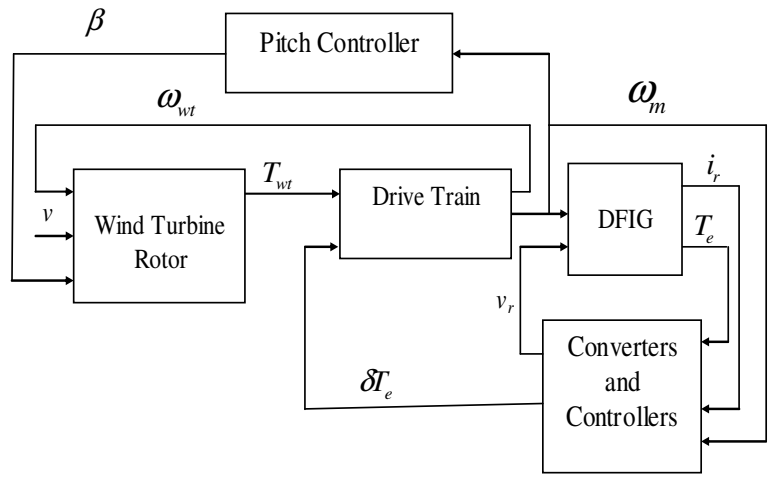

Fig. 2. Block diagram for the control of the mechanical part of 5MW wind turbine in a strong wind. $v_{r}$ and $i_{r}$ are voltage and current of the DFIG rotor respectively.

\section{Designing robust sensor based FDI}

To simplify the steps of designing robust sensor based FDI, we assume all actuators and components are fault free. Equations (19) can then be simplified to:

$$
\dot{x}=A(x) x+B(u) u+d, \quad y=C x+R_{2} f
$$

The residual generator for each sensor will be:

$$
r_{k}=Q_{s k}\left[\left(C e+R_{2} f\right)\left(C_{m}-C_{k}\right)\right]
$$

where $k$ is the number of the measurement sensor; $C_{k}$ is obtained from the matrix $C$ by assuming the $k_{\mathrm{th}}$ row equal zero. $C_{m}$ is equal to the matrix $C$.

From equation (36), it is obvious that each residual generator is driven, so that all other residuals equal zero. From the above, a set of robust and observer-based sensor fault isolation schemes were designed, as illustrated in Fig. 3. Each sensor residual $\left(r_{k}\right)$ is separated from the output of the residual $(r)$ by $r \times\left(C_{m}-C_{k}\right)$, and then dimension of $r_{k}$ is modified using $Q_{s k}$.

Compared to unknown input observers, this scheme is more relaxed. In this approach, instead of decoupling state estimations from unknown inputs, the residual signal is made independent of unknown inputs such as the effective wind speeds on the blades of a wind turbine.

In practice, it was insufficient to identify faults based on residual curves because the system was non-linear, and it worked at different operating points; it was necessary to design a dynamic threshold for each sensor taking into account individual sensor accuracy, supplied by the manufacturer. Therefore, residuals were tested using an interval test dynamic threshold (see Fig. 3). This test gave an output of one (no sensor faults occurred) if the input of the measurement output signals did not exceed the absolute value of the sum of the estimated value, sensor accuracy and sensor noises. The test gave an output of zero (case of sensor fault) if the input of the measurement output signal exceeded the absolute value of the sum of the estimated value, sensor accuracy and sensor noises. The mathematical model of an interval test dynamic block output is based on equation:

$$
\begin{aligned}
& y_{k}(t) \leq\left|\hat{y}_{k}(t)+\delta y_{k}(t)+\eta_{k}\right| \Rightarrow f_{k}(t)=1 \text { fault free } \\
& y_{k}(t)>\left|\hat{y}_{k}(t)+\delta y_{k}(t)+\eta_{k}\right| \Rightarrow f_{k}(t)=0 \text { fault }
\end{aligned}
$$

where $y_{k}(t)$ is a measurement output signal; $\hat{y}_{k}(t)$ estimated value; $\eta_{k}$ is sensor accuracy; and $\delta y_{k}(t)$ is sensor noise.

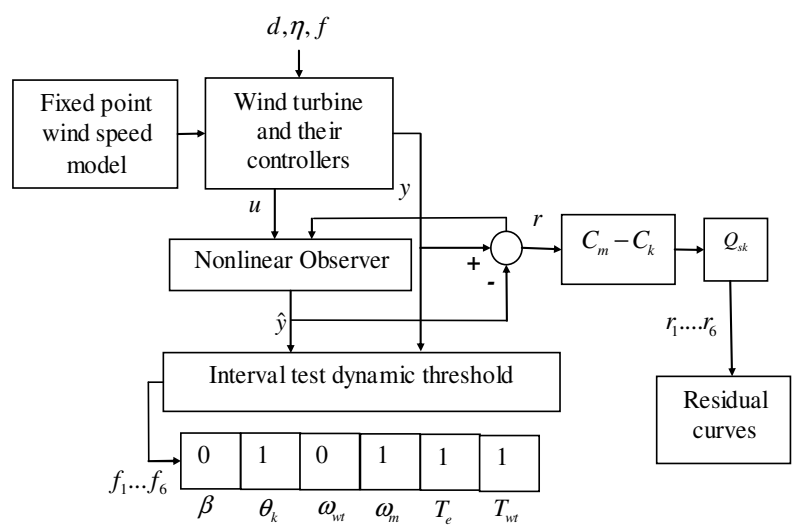

Fig. 3. Robust nonlinear observer-based fault detection and isolation scheme. $d, \eta, f$ are disturbance, sensor noise and sensor fault signals respectively. Here, is indicated " 0 " in the display of Pitch angle and turbine speed sensor faults happened.

Fixed point wind speed model is modelled as a non stationary random process which, as shown in equation (37), is generated by two components (low frequency component $\left(v_{s}\right)$ and turbulence component $\left.\left(v_{t}\right)\right)$ [3].

$$
v(t)=v_{s}(t)+v_{t}(t)
$$

\section{SIMULATION RESULTS}

A nonlinear observer-based residual generator was designed, as shown in Fig. 3. In order to assess the performance of the method, different types of additive sensor fault were applied. The fault types were pitch angle, the difference between turbine rotor angle and generator rotor angle, wind turbine rotor speed, generator rotor speed, electric torque and wind turbine torque. In all simulation cases, we assumed that disturbance and sensor noises are present.

For example, from the behaviour of the residuals curves in Fig. 4, where both wind turbine and electric torque faults occurred at the same time. Here, first the turbine rotor torque sensor apparent and, then, after a few seconds, the electric torque, with its slow response, becomes apparent. In the case of multi-malfunction, the result of the simulation demonstrated that detection and isolation are achieved, as illustrated in Fig. 5 when more than one sensor fault occurred $\left(10 \% \beta, 10 \% \theta_{k}, 10 \% \omega_{w t}, 9 \% \omega_{m}, 10 \% T_{e}\right.$ at the time $250 \mathrm{~s}$, $220 \mathrm{~s}, 200 \mathrm{~s} 180 \mathrm{~s}$ and $150 \mathrm{~s}$, respectively for an effective wind speed of $12 \mathrm{~m} / \mathrm{s}$ ). For example, Fig. 5 clearly illustrates the robustness of this method when compared to FDI. It demonstrates that as pitch angle and rotor speed sensor faults occur, they are represented as zeroes in the fault display, as shown in Fig. 3. 

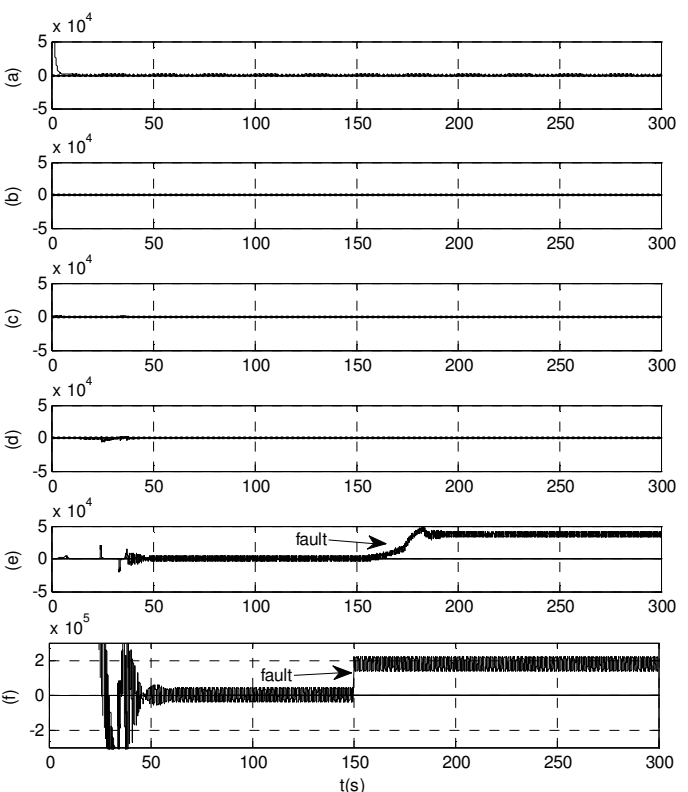

$\mathrm{t}(\mathrm{s})$

Fig. 4. Residual norms when an $8 \%$ wind rotor torque and a $10 \%$ electric torque sensor fault occur at time-point $(t)=150 \mathrm{~s}$ with an effective wind speed of $12 \mathrm{~m} / \mathrm{s}$ for (a) pitch angle, (b) difference between turbine rotor angle and generator rotor angle, (c) wind turbine rotor speed, (d) generator rotor speed, (e) electric torque and (f) wind turbine torque
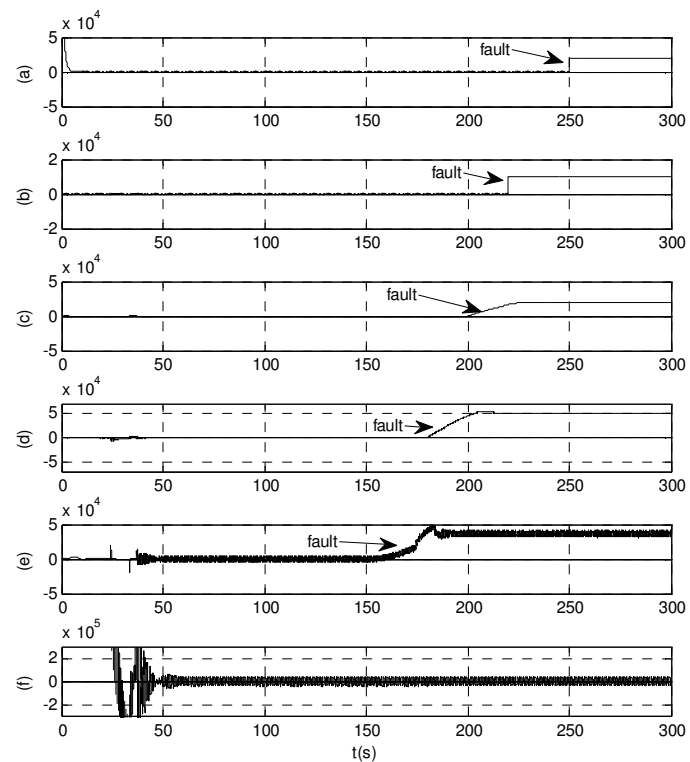

Fig. 5. Residual norms for $10 \%$ pitch angle, 0.1 difference between turbine rotor angle and generator rotor angle, 10\% wind turbine rotor speed, $9 \%$ generator rotor speed and $10 \%$ electrical torque sensor faults occurring at time-points $(\mathrm{t})=250,220,200,180$ and $150 \mathrm{~s}$, respectively, with an effective wind speed of $12 \mathrm{~m} / \mathrm{s}$ for (a) pitch angle, (b) difference between turbine rotor angle and generator rotor angle, (c) wind turbine rotor speed, (d) generator rotor speed, (e) electric torque and (f) wind turbine torque

\section{CONCLUSIONS}

State-dependent nonlinear observer-based scheme is developed for early FDI in wind turbine rotor, drive-train and generator sensors. A nonlinear observer is designed using state dependent differential Riccati equation rather than algebraic Riccati equations, which can require an overly restrictive requirement on the observability and controllability of the system. This paper is aimed at detecting faults in wind turbine's sensors. Therefore, The fault detection system is designed and optimized to be most sensitive to system faults and least sensitive to system disturbances and noises. The residual generator based on the nonlinear observer is employed to develop a monitoring system. Simulation results illustrated that the proposed method is a robust method to detect faults in sensors of the wind turbine.

The advantages of this scheme are that it is systematic to design and implement the algorithm in a practical way. Also, by using return of ' 1 ' for each non-faulty sensor and ' 0 ' for a faulty sensor, both single and multiple faults can be located quickly. In this approach, instead of decoupling state estimations from unknown inputs, the residual signal is made independent of unknown inputs such as the effective wind speeds on the blades of a wind turbine.

\section{REFERENCES}

[1] A. Hwas, and R. Katebi, "Model-based fault detection and isolation for wind turbine," Control (CONTROL), 2012 UKACC International Conference, pp. 876-881.

[2] D. A. Haessig, and B. Friedland, "State dependent differential Riccati equation for nonlinear estimation and control," in Proc. 15th Triennial World Cong., arcelona, Spain, 2002, IFAC.

[3] A. Hwas, "Wind energy conversion system model," Industrial Control Centre, technical progress report first year, University of Strathclyde, 2010.

[4] G. Feng, X. Daping, and L. Yuegang, "Pitch-control for Large-scale Wind Turbine Based on Feed forward Fuzzy," PI. WCOICA, 25-27, Chongqing, China, 2008.

[5] C. Eisenhut, F. Krug, and C. Schram, "Wind-Turbine Model for System Simulations Near Cut-In Wind Speed," IEEE Transactions on Energy Conversion, vol (22), NO.2, 2007.

[6] M.H. Hansen, A. Hansen, T.J. Larsen, S. Seoye, P. Seoensen and P. Fuglsang, "Control design for a pitch-regulated, variable speed wind turbine, " technical report, Riseo National Laboratory, 2005.

[7] A.D. Hansen, P. Soerensen, F. Blaabjerg, and J. Becho, "Dynamic modelling of wind farm grid interaction," Wind Engineering, 26(4):191-208, 2002.

[8] Z. Lubosny, "Wind Turbine Operation in Electric Power Systems," Springer- erlag, Berlin, 2003.

[9] Y. Zhao, X.D. Zou, Y.N Xu, Y. Kang and J. Chen, "Maximal Power Point tracking under speed-mode control for wind energy generation system with doubly fed introduction generator," Power Electronics and Motion Control Conference, 2006, IPEMC 2006, CES/IEEE 5th International, vol. 1, pp.1-5, Aug 2006.

[10] J. Chen, and R.J. Patton, "Robust model-based fault diagnosis for dynamic systems," Kluwer Academic Publishers, 1999.

[11] K. Johnson, L. Pao, M. Balas, and L. Fingersh, "Control of variablespeed wind turbines: standard and adaptive techniques for maximizing energy capture," IEEE Control Systems Magazine, 26(3):70-81, 2006.

[12] A. Hwas, and R. Katebi, "Wind turbine control using PI pitch angle controller," IFAC Conference on Advances in PID Control PID'12, March 2012

[13] I. Munteanu, A. Bratcu, N. Cutulis, and E. Ceanga, "Optimal control of wind energy system," Springer, London, 2008.

[14] N. Heraud, M.A. Kahyeh, and I.S. Guelle, "Monitoring of the DFIG of a variable speed wind turbine," in Transmission and Distribution Conference and Exposition, Latin America (T\&D-LA), IEEE/PES, 2010.

[15] S.P. Azad, and J.E. Tate, "Parameter estimation of doubly fed induction generator driven by wind turbine,". in Power Systems Conference and Exposition (PSCE), IEEE/PES. 2011. 